\title{
Peripheral artery disease risk factors in Jeddah, Saudi Arabia: a retrospective study
}

\author{
Abdulrahman Ibrahim Mufti \\ Alsadiqi \\ Ahmed Hussein Subki \\ Rami Hussam Abushanab \\ Mohammed Rashid \\ Ocheltree \\ Hattan Ahmed Bajahmom \\ Yosef Ibrahim Mufti Alsadiqi \\ Wesam Awad Alhejily
}

Department of Internal Medicine, College of Medicine, King Abdulaziz University, Jeddah, Saudi Arabia
Correspondence: Wesam Awad Alhejily Department of Internal Medicine, College of Medicine, King Abdulaziz University, PO Box 43129, Jeddah 2156I, Saudi Arabia

Tel +966546033322

Email walhejily@kau.edu.sa
This article was published in the following Dove Press journal: International Journal of General Medicine

Background: Peripheral artery disease (PAD) is a major health problem in Saudi Arabia with considerable implications for morbidity and mortality. Many risk factors have been established for developing PAD, but the prevalence of these risk factors is variable from one country to another. Aim: To identify the risk factors for PAD and study their prevalence in Jeddah, Saudi Arabia. Methods: A retrospective chart review study was conducted in a tertiary center in Jeddah from July 2012 to September 2015. All patients with PAD were recruited into this study, and their data were analyzed using IBM SPSS. Correlation between PAD and various risk factors was studied using Spearman's coefficient.

Results: A total of 261 patients were recruited, of which 55\% were males. Hypertension, diabetes type 2 , and obesity were found among $34.2 \%, 33.3 \%$, and $29.2 \%$ of the patients, respectively. About $45 \%$ had a history of previous vascular surgery, and amputation was performed in $6.9 \%$ particularly in legs. Hypertension and metabolic syndrome were the only risk factors that showed a significant correlation with $\mathrm{PAD}(P=0.039$ and 0.040 , respectively).

Conclusion: The most prevalent risk factors for PAD in Jeddah were hypertension, diabetes, obesity, and smoking. Hypertension and metabolic syndrome were the only risk factors significantly associated with PAD. Males were often more affected by PAD than females. The most commonly affected site was the lower limbs.

Keywords: prevalence, peripheral artery disease, risk factors, Saudi Arabia

\section{Introduction}

Peripheral artery disease (PAD) is a major health problem affecting around 27 million individuals in the Western countries and $\sim 12 \%$ of Saudi population. ${ }^{1,2}$ PAD is part of a widespread systemic process characterized by atherosclerosis of arterial walls of peripheral arteries (ie, all arteries outside the heart) in various organs resulting in ischemia which might require subsequent amputation. PAD is commonly associated with coronary artery disease (CAD) and cerebrovascular disease as they share common pathophysiological characteristics, risk factors, and management lines. ${ }^{3}$ The pathophysiological mechanism of PAD comprises the formation of intraarterial atheroma consisting of a cholesterol core and fibrous coat resulting in insidious progressive occlusion of medium- and large-sized arteries all over the body. PAD is typically segmental, and thus, the clinical presentation varies significantly from one patient to another. ${ }^{4}$

Because PAD carries considerably high rates of morbidity and mortality, it is essential to implement all possible strategies to prevent and treat the disease. Successful 
prevention requires accurate identification of risk factors and tight measures to control them. Over many decades, various risk factors for PAD have been studied and are well established. It has been identified that smoking is the most potent risk factor for the disease, and that it can increase an individual's risk for peripheral ischemia up to seven times. ${ }^{5}$ Diabetes mellitus, hypertension, and dyslipidemia are the risk factors that come next with their negative influence on arterial walls. ${ }^{3,6}$ Chronic kidney disease and male gender are reported to increase the risk for PAD, and other less established risk factors include high C-reactive protein level, inflammation, elevated fibrinogen level, and increased blood lipoprotein (a) level. ${ }^{7,8}$

For cost-effective prevention of a disease, each country needs to identify its risk factors and their prevalence. Identification of risk factors for PAD that are specific for Saudi Arabia will allow the healthcare authorities to establish suitable preventive measures according to the burden and prevalence of the local risk factors. Therefore, the aim of this research was to identify the various risk factors for PAD and study their prevalence in Saudi Arabia and to explore the correlation of these risk factors to the disease.

\section{Patients and methods}

To achieve the research aim, a retrospective chart review study was conducted at the teaching hospital affiliated to King Abdulaziz University (KAUH) in Jeddah, Saudi Arabia. Medical records during the period from July 2012 to September 2017 were reviewed, and all patients diagnosed with PAD were considered for recruitment into this study. PAD was diagnosed with clinical examination (weak or absent pulses), measurement of the ankle-brachial index $(<0.9)$, and duplex ultrasonography. ${ }^{9}$ The diagnosis was made by general surgery consultants, that is, assistant professors, associate professors, or professors, at KAUH. Patients with PAD at the neck, upper limbs, lower limbs, liver, intestine, or kidneys were recruited. Any patients with PAD or with a history of PAD in the past 5 years with or without hypertension, diabetes mellitus type 1 or 2 , metabolic syndrome, or heart failure were also included in this study. Hypertension was diagnosed by senior internal medicine consultants according to the 2017 American College of Cardiology guidelines for hypertension in adults. ${ }^{10}$ Metabolic syndrome was diagnosed when a patient had at least three of the following: increased waist circumference (more than 40 inches in males or more than 35 inches in females), triglycerides above $150 \mathrm{mg} / \mathrm{dL}$, fasting blood glucose level higher than $100 \mathrm{mg} / \mathrm{dL}$, high-density lipoprotein cholesterol below $50 \mathrm{mg} / \mathrm{dL}$ in females or $40 \mathrm{mg} / \mathrm{dL}$ in males, or SBP above $130 \mathrm{mmHg}$ or DBP above $85 \mathrm{mmHg}$.

All demographic, clinical, laboratory, and imaging data were fed to the computer and analyzed using IBM SPSS software version 20.0 (IBM, Armonk, NY, USA). There were no missing data in the collected data set, and therefore, missing data management was not required. Discrete variables were described using frequencies and percentages, whereas continuous variables were presented using mean and SD. The associations between PAD located in the leg(s) and multiple variables including gender, nationality, obesity, hypertension, smoking, diabetes mellitus type 1 and 2, metabolic syndrome, $\mathrm{CAD}$, heart failure, previous surgery, previous catheterization, and family history of PAD were explored using chi-squared test. The relationship between PAD located in the leg(s) and age of the patients was evaluated by independent samples $t$-test. The significance of the analyzed data was judged at the 95\% CI and 5\% probability level.

This study was approved by the Unit of Biomedical Ethics Research Committee at King Abdulaziz University, and it was conducted in accordance with the Declaration of Helsinki. Patients' consent to review their medical records was not required by the ethics committee; this waiver was due to the retrospective chart review nature of our study. No personal data that could be linked to a patient were used. Patient data confidentiality was strictly protected when conducting this study.

\section{Results}

A total of 240 patients with PAD were included in this retrospective chart review study. The mean age of all cases was $58.61 \pm 18.35$ years, and $55.8 \%$ of them were males. About half of the recruited patients (52.9\%) were native Saudi, while the remaining half were of different nationalities. The mean height, weight, and body mass index (BMI) of all patients were $161.74 \pm 14.08 \mathrm{~cm}, 74.47 \pm 22.75 \mathrm{~kg}$, and $28.24 \pm 8.11 \mathrm{~kg} /$ $\mathrm{m}^{2}$, respectively (Table 1 ). The most common risk factors encountered among patients with PAD were "hypertension" which was found in $34.2 \%$ of cases and "diabetes mellitus type 2 " found in $33.3 \%$ of cases. Around $45.0 \%$ of patients had a history of previous vascular surgery. The other risk factors and their prevalence rates are detailed in Table 2.

With regard to the laboratory tests, the mean $\pm \mathrm{SD}$ of $\mathrm{HbAlc}$, platelet count, prothrombin time, partial thromboplastin time, and international normalized ratio among all patients were $7.69 \% \pm 2.19 \%, 280.73 \pm 122.46 \times 10^{9} / \mathrm{L}, 16.93 \pm 10.55$ seconds, $38.56 \pm 15.43$ seconds, and $1.54 \pm 1.12$ (Table 3 ). The most common site for PAD was the legs $(63.8 \%)$, and the least 
Table I Baseline characteristics of all included patients $(n=240)$

\begin{tabular}{|l|l|}
\hline Characteristics & N (\%) \\
\hline Age in years (mean $\pm S D)$ & $58.61 \pm 18.35$ \\
Gender & \\
- Male & $134(55.8)$ \\
- Female & $106(44.2)$ \\
Nationality & \\
- Saudi & $127(52.9)$ \\
- Non-Saudi & $113(47.1)$ \\
Height in $\mathrm{cm}(\operatorname{mean} \pm \mathrm{SD})$ & $161.74 \pm 14.08$ \\
Weight in $\mathrm{kg}(\operatorname{mean} \pm \mathrm{SD})$ & $74.47 \pm 22.75$ \\
Body mass index in $\mathrm{kg} / \mathrm{m}^{2}($ mean $\pm S D)$ & $28.24 \pm 8.11$ \\
\hline
\end{tabular}

Table 2 Prevalence of risk factors of PAD in all patients presented in descending order $(n=240)$

\begin{tabular}{|l|l|l|l|}
\hline Risk factor & N & $\%$ & P-value \\
\hline Previous vascular surgery & 108 & 45.0 & 0.756 \\
Hypertension & 82 & 34.2 & 0.039 \\
Diabetes mellitus type 2 & 80 & 33.3 & 0.776 \\
Obesity & 70 & 29.2 & 0.172 \\
Diabetes mellitus type I & 25 & 10.4 & 0.074 \\
Previous catheterization & 23 & 9.6 & 0.225 \\
Smoking & 21 & 8.8 & 0.444 \\
Heart failure & 16 & 6.7 & 0.235 \\
Coronary artery disease & 14 & 5.8 & 0.085 \\
Metabolic syndrome & 5 & 2.1 & 0.040 \\
Family history of PAD & $\mathrm{I}$ & 0.4 & 0.184 \\
\hline
\end{tabular}

Abbreviation: PAD, peripheral artery disease.

Table 3 Distribution of all patients by blood test parameters $(n=240)$

\begin{tabular}{|l|l|l|l|l|l|}
\hline Parameter & Mean & SE & \pm SD & Min & Max \\
\hline HbAIc & 7.69 & 0.20 & \pm 2.19 & $4 . I I$ & 15.00 \\
Platelet count & 280.73 & 7.92 & $\pm I 22.46$ & 40.00 & 768.00 \\
PT & 16.93 & 0.69 & \pm 10.55 & 9.90 & 91.60 \\
PTT & 38.56 & 1.00 & \pm 15.43 & 11.70 & 132.00 \\
INR & 1.54 & 0.07 & $\pm I .12$ & 0.80 & 9.50 \\
\hline
\end{tabular}

Abbreviations: INR, international normalized ratio; Max, maximum; Min, minimum; PT, prothrombin time; PTT, partial thromboplastin time.

common was the kidneys as renal PAD was observed only in a single patient $(0.4 \%)$; this patient with renal PAD was not reported to have fibromuscular dysplasia. The distribution of PAD according to the affected site is displayed in Table 4. Most of the PAD cases were diagnosed by ultrasonography (64.2\%). Computed tomography angiogram was used to diagnose $38.8 \%$ of cases, and only three patients $(1.3 \%)$ underwent exercise stress test to diagnose PAD (Table 4). Many medications were used for treatment of PAD among the included patients such as clopidogrel, aspirin, and enoxaparin. Clopidogrel was used in $31.7 \%$ of cases, aspirin was used in
Table 4 Distribution of all included patients by PAD site, tests used to diagnose PAD (ultrasonography, CT angiogram, exercise stress test), amputation, and drugs used for the treatment of PAD (Plavix, aspirin, enoxaparin) $(n=240)$

\begin{tabular}{|l|l|l|}
\hline Characteristics & N & $\%$ \\
\hline Site of PAD & & \\
- Arm & 2 & 0.8 \\
- Intestine & 13 & 5.4 \\
- Kidney & $\mathrm{I}$ & 0.4 \\
- Leg & 153 & 63.8 \\
- Liver & 36 & 15.0 \\
- Neck & 35 & 14.6 \\
- Ultrasonography & & \\
- CT angiogram & 154 & 64.2 \\
- Exercise stress test & 93 & 38.8 \\
Amputation & 3 & 1.3 \\
Drug use & 18 & 6.9 \\
- Clopidogrel & & \\
- Aspirin & 76 & 31.7 \\
- Enoxaparin & 124 & 51.7 \\
\hline
\end{tabular}

Notes: ${ }^{a} n=250$ because more than one test was done on a few patients. ${ }^{b} n=358$ because a combination of two or three drugs was used in a few patients.

Abbreviations: CT, computed tomography; PAD, peripheral artery disease. respectively

$51.7 \%$ cases, and enoxaparin was prescribed to $65.8 \%$ of cases (Table 4). The medications studied were those given during the patients' stay as inpatients. When the leg PAD was assessed separately, it was found that a higher percentage $(70.6 \%)$ of cases were diagnosed with ultrasonography. For PAD of the $\operatorname{leg}(\mathrm{s})$, enoxaparin was the most common drug prescribed; it was used by $68.6 \%$ of patients (Table 5). Among the included patients, a total of $18(6.9 \%)$ required leg amputation due to severe PAD; 16 (88.9\%) of these patients had PAD only in the lower extremities, and only two patients had associated neck or intestinal ischemia (Table 6).

In this research, the association between PAD of the leg(s) and various demographic characteristics and risk factors was studied. The variables studied were age, gender, nationality, smoking, obesity, hypertension, diabetes mellitus type 1 and 2, metabolic syndrome, cerebrovascular accident, CAD, heart failure, previous surgery, previous catheterization, and family history of PAD. The presence of hypertension $(P=0.039)$ and metabolic syndrome $(P=0.040)$ was statistically significantly associated with PAD of the leg(s), whereas the other studied risk factors were not significantly associated with PAD of the $\operatorname{leg}(\mathrm{s})(P$-values $>0.05)$ (Table 2$)$.

\section{Discussion}

PAD was reported to affect about $12 \%$ of population in Saudi Arabia. ${ }^{2}$ In this study, all patients with PAD admitted at a tertiary center in Jeddah during the period from July 2012 
Table 5 Distribution of patients with PAD in the leg(s) by diagnostic tests used and drugs used for treatment of PAD $(n=153)$

\begin{tabular}{|l|l|l|}
\hline Characteristics & N & $\%$ \\
\hline Tests used to diagnose PAD & & \\
- Ultrasonography & 108 & 70.6 \\
- CT angiogram & 63 & 41.2 \\
- Exercise stress test & 3 & 2.0 \\
\hline Drugs used for treatment of PAD & & \\
- Clopidogrel & 49 & 32.0 \\
- Aspirin & 75 & 49.0 \\
- Enoxaparin & 105 & 68.6 \\
\hline
\end{tabular}

Abbreviations: CT, computed tomography; PAD, peripheral artery disease.

Table 6 Distribution of frequency of leg amputation by PAD sites $(n=18)$

\begin{tabular}{|l|l|}
\hline PAD site & N (\%) \\
\hline Leg & $16(88.9)$ \\
\hline Neck & I (5.6) \\
Intestine & I (5.6) \\
\hline
\end{tabular}

Notes: Total number of leg amputations was 18. No cases required amputation when PAD was in a location other than the leg(s), neck, and intestine.

Abbreviation: PAD, peripheral artery disease.

to September 2017 were studied to explore the prevalence of risk factors among PAD patients in Saudi Arabia, and to determine the correlation between these factors and the disease. The different sites of PAD were studied, but a special focus was given to the lower limb PAD.

In agreement with most of the literature studies, the present study showed that the majority of patients with PAD were males (58\%). ${ }^{9-13}$ Male gender was previously identified as a risk factor for PAD because males lack the hormones that protect against atherosclerosis. Estrogen and progesterone are thought to be responsible for the lower prevalence of PAD in premenopausal females. Estrogen is known to improve the degradation of low-density lipoprotein cholesterol, to suppress the proliferation of the tunica media of the blood vessel wall, and to inhibit extracellular matrix production. Furthermore, estradiol inhibits platelet aggregation. Progesterone also plays an important anti-atherosclerotic role both directly on the blood vessel wall and indirectly via potentiating the antioxidant action of estrogen. ${ }^{14,15}$ In few studies, females had a higher prevalence of PAD and this was attributed by the researchers to the older age and the other associated risk factors among the studied females. ${ }^{16,17}$ The effect of age on PAD could not be evaluated in this study due to our study design which only included a limited number $(n=261)$ of PAD patients.
Almost half (45.2\%) of the patients with PAD recruited into this study had a history of previous vascular surgery. This might indicate the severity of PAD in Saudi patients necessitating surgery. Previous catheterization was found in only $10 \%$ of patients. These numbers were higher than the number of patients requiring interventional management in literature that was estimated to be around $18 \% .{ }^{18}$ This may be attributed to the different population characteristics and different prevalence of various risk factors among the Saudi population. Another indicator of severity in the sample studied in this research was the slightly high prevalence $(6.9 \%)$ of amputations specifically for the PAD of the lower limb(s). The average rate of lower limb amputation complicating PAD reported in the literature was $5 \% .{ }^{19}$ However, this could also be due to selection bias, with more severe cases coming to a tertiary center.

As regards the medication used for treatment of PAD, data from this study showed that Saudi physicians preferred to prescribe a therapeutic anticoagulant (enoxaparin) to aspirin or clopidogrel in more than two-thirds of the patients. Though antithrombotic agents are recommended as the firstline therapy by both the American and European guidelines for management of PAD, the high prevalence of associated comorbidities necessitating anticoagulation (such as CAD, heart failure, and so on) may be the reason for prescribing anticoagulants among the vast majority of the studied patients. $^{20,21}$

Hypertension and diabetes mellitus type 2 was found in almost one-third of the recruited patients. Hypertension promotes fatty streak deposition on the endothelial lining of blood vessels leading to atherosclerotic plaque formation and progression, and endothelial dysfunction. ${ }^{22}$ Additionally, hypertensive shearing forces lead to plaque rupture. Therefore, hypertension has been reported to be significantly associated with PAD in $33 \%-55 \%$ of the cases. ${ }^{23}$ This study also showed that hypertension was significantly correlated with PAD with a $P$-value of 0.028 . Similarly, diabetes mellitus-associated hyperglycemia results in impairment of vascular repair mechanisms, alteration of the ROS generation, and changes in platelet reactivity and coagulation pathway. ${ }^{24}$ Consequently, diabetes increases an individual's risk for PAD by two- to fourfold. ${ }^{25}$ Furthermore, it enhances the disease somatization; about $20 \%$ of patients with PAD were reported to have diabetes. ${ }^{3}$ A promising finding noted in this study is that the mean HbA1c of the included patients was at a fairly controlled level, which reflects proper health education and patient care. 
Obesity was found in more than one-fourth of the patients in this study, and the mean BMI of the patients in this study $\left(27.93 \pm 7.99 \mathrm{~kg} / \mathrm{m}^{2}\right)$ was in the overweight range. Metabolic syndrome was found only in a minority of the included patients (1.9\%). Similar to these results, Huang et al reported that each $0.43 \mathrm{~kg} / \mathrm{m}^{2}$ increase in BMI was associated with an OR of 1.17 for PAD $(P<0.0004) .{ }^{26}$ They also found a causeeffect relationship between PAD and obesity $(P<0.0003)$. Similar data were reported in other studies. ${ }^{27-29}$

Smoking is a well-established risk factor for PAD. In this study, only $8.8 \%$ of patients were smokers. This may be due to the Islamic cultural background which prohibits this habit and the different culture. Smoking has been reported to increase the risk for PAD up to sevenfold. ${ }^{5} \mathrm{~A}$ review article has indicated that the OR for PAD in smokers ranged from 1.5 to $4.2 .^{3}$

Heart failure (6.5\%) and CAD (14.2\%) were found in a minority of PAD cases included in this research. In disagreement with these findings, many researchers reported an overlap between PAD and one or both of these diseases in more than $20 \%$ of the patients. ${ }^{28,29}$ This high degree of overlap is often attributed to the common underlying pathophysiological mechanisms that result in progressive atherosclerosis of medium- and large-sized blood vessels. ${ }^{16,25}$ Though the association between cerebrovascular accident and PAD was not significant in the patients studied, cerebrovascular accident was significantly correlated with PAD as shown by regression analysis $(P<0.0001)$.

One of the main limitations of this study was that data on many of the important variables (such as blood pressure, biochemical lipid parameters, renal function, and so on) were not available in the records, and therefore, could not be gathered and analyzed. Selection bias might be another limitation to our study because KAUH is a tertiary referral center at which most of the cases of PAD had a history of previous vascular surgery. Future prospective, multicenter studies are needed to assess the prevalence of risk factors among patients with PAD, and those factors that were missing in the records should be included and studied.

\section{Conclusion}

The most prevalent risk factors for PAD in Jeddah were hypertension, diabetes, and obesity. The only factors that significantly correlated with PAD were cerebrovascular accident and hypertension. Males were more affected by PAD than females, and the lower limbs were the site most commonly affected by PAD. The rates of lower limb amputation were slightly higher than those reported in literature.

\section{Acknowledgments}

The authors thank Abdullah Yasir Aljohani, Sara Nawfal Sharief, Nada Abdullatif Hefni, Ali Omar Hussein, and Aseel Abdu aljahdali for their help in data collection.

\section{Author contributions}

All authors contributed to data analysis, drafting or revising the article, gave final approval of the version to be published, and agree to be accountable for all aspects of the work.

\section{Disclosure}

AIMA and YIMA are brothers. The authors report no other conflicts of interest in this work.

\section{References}

1. Peach G, Griffin M, Jones KG, Thompson MM, Hinchliffe RJ. Diagnosis and management of peripheral arterial disease. BMJ. 2012;345:e5208.

2. Al-Sheikh SO, Aljabri BA, Al-Ansary LA, Al-Khayal LA, Al-Salman MM, Al-Omran MA. Prevalence of and risk factors for peripheral arterial disease in Saudi Arabia. A pilot cross-sectional study. Saudi Med J. 2007;28(3):412-414.

3. El-Menyar A, Al Suwaidi J, Al-Thani H. Peripheral arterial disease in the Middle East: underestimated predictor of worse outcome. Glob Cardiol Sci Pract. 2013;2013(2):13.

4. Stephens E. Peripheral Vascular Disease. Available form: https:// emedicine.medscape.com/article/761556-overview. Accessed Deecember 24, 2018.

5. Heliövaara M, Karvonen MJ, Vilhunen R, Punsar S. Smoking, carbon monoxide, and atherosclerotic diseases. Br Med J. 1978;1(6108): $268-270$.

6. Emerging Risk Factors Collaboration, Sarwar N, Gao P, et al. Diabetes mellitus, fasting blood glucose concentration, and risk of vascular disease: a collaborative meta-analysis of 102 prospective studies. Lancet. 2010;375(9733):2215-2222.

7. Emerging Risk Factors Collaboration, Kaptoge S, Di Angelantonio E, et al. C-reactive protein, fibrinogen, and cardiovascular disease prediction. N Engl J Med. 2012;367(14):1310-1320.

8. Emerging Risk Factors Collaboration, Di Angelantonio E, Sarwar N, et al. Major lipids, apolipoproteins, and risk of vascular disease. JAMA. 2009;302(18):1993-2000.

9. Hennion DR, Siano KA. Diagnosis and treatment of peripheral arterial disease. Am Fam Physician. 2013;88(5):306-310.

10. Whelton PK, Carey RM, Aronow WS, et al. ACC/AHA/AAPA/ $\mathrm{ABC} / \mathrm{ACPM} / \mathrm{AGS} / \mathrm{APhA} / \mathrm{ASH} / \mathrm{ASPC} / \mathrm{NMA} / \mathrm{PCNA}$ guideline for the prevention, detection, evaluation, and management of high blood pressure in adults: a report of the American College of Cardiology/ American Heart Association Task Force on Clinical Practice Guidelines. Hypertension. 2017;71:e13-e115.

11. Egorova N, Vouyouka AG, Quin J, et al. Analysis of gender-related differences in lower extremity peripheral arterial disease. J Vasc Surg. 2010;51(2):372-378.

12. Rowe VL, Lee W, Weaver FA, Etzioni D. Patterns of treatment for peripheral arterial disease in the United States: 1996-2005. JVasc Surg. 2009;49(4):910-917.

13. Teodorescu VJ, Vavra AK, Kibbe MR. Peripheral arterial disease in women. J Vasc Surg. 2013;57(4 Suppl):18S-26S.

14. Price J, Leng GC. Steroid sex hormones for lower limb atherosclerosis. Cochrane Database Syst Rev. 2012;10:CD000188.

15. Kumakura H, Kanai H, Araki Y, et al. Sex-related differences in Japanese patients with peripheral arterial disease. Atherosclerosis. 2011;219(2):846-850. 
16. Kalbaugh CA, Kucharska-Newton A, Wruck L, et al. Peripheral artery disease prevalence and incidence estimated from both outpatient and inpatient settings among medicare fee-for-service beneficiaries in the Atherosclerosis Risk in Communities (ARIC) study. J Am Heart Assoc. 2017;6(5):e003796.

17. Hiramoto JS, Katz R, Weisman S, Conte M. Gender-specific risk factors for peripheral artery disease in a voluntary screening population. $\mathrm{J} \mathrm{Am}$ Heart Assoc. 2014;3(2):e000651.

18. Andreozzi GM, Martini R. The fate of the claudicant limb. Eur Heart J. 2002;4:B41-B45.

19. Swaminathan A, Vemulapalli S, Patel MR, Jones WS. Lower extremity amputation in peripheral artery disease: improving patient outcomes. Vasc Health Risk Manag. 2014;10:417-424.

20. Members AF, Aboyans V, Ricco J-B. Editor's choice - 2017 ESC guidelines on the diagnosis and treatment of peripheral arterial diseases, in collaboration with the European Society for Vascular Surgery (ESVS). Eur J Vasc Endovasc Surg. 2018;55(3):305-368.

21. Gerhard-Herman MD, Gornik HL, Barrett C. AHA/ACC guideline on the management of patients with lower extremity peripheral artery disease: executive summary. Vas Med. 2017;22:NP1-NP43.

22. Zaheer M, Chrysostomou P, Papademetriou V. Hypertension and atherosclerosis: pathophysiology, mechanisms and benefits of BP control. In: Andreadis EA, editor. Hypertension and Cardiovascular Disease; 2016:201-216.
23. Makin A, Lip GY, Silverman S, Beevers DG. Peripheral vascular disease and hypertension: a forgotten association? J Hum Hypertens. 2001;15(7):447-454

24. Paneni F, Beckman JA, Creager MA, Cosentino F. Diabetes and vascular disease: pathophysiology, clinical consequences, and medical therapy: part I. Eur Heart J. 2013;34(31):2436-2443.

25. Newman AB, Siscovick DS, Manolio TA, et al. Ankle-arm index as a marker of atherosclerosis in the Cardiovascular Health Study. Cardiovascular Heart Study (CHS) Collaborative Research Group. Circulation. 1993;88(3):837-845.

26. Huang $\mathrm{Y}, \mathrm{Xu} \mathrm{M}, \mathrm{Xie} \mathrm{L}$, et al. Obesity and peripheral arterial disease: a Mendelian randomization analysis. Atherosclerosis. 2016;247: 218-224.

27. Brouwer BG, Visseren FL, Stolk RP, van der Graaf Y, SMART Study Group. Abdominal fat and risk of coronary heart disease in patients with peripheral arterial disease. Obesity. 2007;15(6):1623-1630.

28. Ouriel K, Disease Parterial. Peripheral arterial disease. Lancet. 2001;358(9289):1257-1264.

29. Fowkes FG, Rudan D, Rudan I, et al. Comparison of global estimates of prevalence and risk factors for peripheral artery disease in 2000 and 2010: a systematic review and analysis. Lancet. 2013;382(9901):1329-1340.
International Journal of General Medicine

\section{Publish your work in this journal}

The International Journal of General Medicine is an international, peer-reviewed open-access journal that focuses on general and internal medicine, pathogenesis, epidemiology, diagnosis, monitoring and treatment protocols. The journal is characterized by the rapid reporting of reviews, original research and clinical studies across all disease areas.

\section{Dovepress}

The manuscript management system is completely online and includes a very quick and fair peer-review system, which is all easy to use. Visit http://www.dovepress.com/testimonials.php to read real quotes from published authors. 